\title{
Relationship Between Management Policies and Profitability of Manufacturing Companies Listed in the First Section of the Tokyo Stock Exchange (2016 Results)
}

\author{
Ryozo Kitajima *, Ryotaro Kamimura $^{\dagger}$, Hiroyuki Sakai ${ }^{\ddagger}$
}

\begin{abstract}
Management policies are important guidelines for companies; they describe the founding spirit, company motto, and other objectives. Therefore, we consider that management policy may affect a company's profitability. This study examined management policies from manufacturing companies and extracted keywords that influence profitability.

Management policies and profitability [measured by the return on assets (ROA)] data were collected from annual securities reports, because the report descriptions are accurate, and the reports are easy to obtain. The management policies were written in natural language, and the analysis data were complex; therefore, we used a neural computational method known as "potential learning," which can interpret internal representations. To extract keywords that influence profitability, we designed a classification model of profitability with the management policy as input, and a model with an accuracy of 0.6115 was created. The results suggest that the management policies of highly profitable companies are diverse in using the word "improvement," which may influence the ROA.
\end{abstract}

Keywords: management policy, profitability, neural network, feature extraction

\section{Introduction}

In this study, we try to extract keywords that influence companies' profitability using the management policies of manufacturing companies listed in the first section of the Tokyo Stock Exchange (TSE) in 2016. Management policy (also known as business philosophy, corporate philosophy, or mission statement [1]) is a guideline to convey matters significant to the business, such as its founding spirit, company motto, and other objectives. Companies profit from daily business activities, and at the end of the fiscal year, their performances are summarized and their annual corporate performance is announced. On this day, companies know the results of their high and low performance, and we consider that these results may be influenced by their management policies. Nevertheless, when evaluating a company's performance practically, it is implausible to assume that management policy alone

* Tokyo Polytechnic University, Kanagawa, Japan

$\dagger$ Tokai University, Kanagawa, Japan

\# Seikei University, Tokyo, Japan 
has a significant impact, because various factors surrounding each company, such as market conditions and capitalization, affect the company's overall performance. However, it is improbable that a company would ignore the business stance and guidelines expressed in the management policy, and, therefore, we cannot ignore that management policies may impact business performance.

Although the relationship between companies' management policies and profitability has been studied previously, no clear results have been reported. For example, Takao [2] stated in a research review that "it is considered that there is sufficient possibility that management philosophies can contribute to corporate earnings." Bart et al. [3] stated in a study targeting Canadian companies that "the presence of mission statements were not automatically associated with superior firm performance."

The remainder of this paper is organized as follows. In section 2, we describe studies related to the relationship between management policies and profitability. In section 3, we explain the flow of the current study; management policies to be collected; data to be analyzed; and the method to be used. In section 4, we discuss our results, and finally, we conclude the paper in section 5 .

\section{Related Work}

There are several studies that analyze textual data transmitted by organizations. For example, Cortés [4] analyzed universities' mission statements and reported the differences such as public universities' emphasis on students and private universities' emphasis on education. Nakagawa et al. [5] also analyzed corporate integrated reports and reported that non-financial information of companies has been attracting attention in recent years and the characteristics of integrated reports that are judged to be "good."

In addition, there are several studies that not only extract the features of organizational statements, but also investigate the relationship between the extracted features and the results (e.g., business performance). For example, several studies have focused on the relationship between a company's declarations, such as management policies, and the company's performance. For example, Patel et al. [6] investigated the relationship between mission statement and organizational performance in non-profit organizations through a questionnaire survey of organizations, and reported that "mission statements have a significant positive relationship with organizational performance." and "organisational commitment, particularly affective commitment, moderates the relationship between mission statements and organisational performance." In addition, Williams et al. [7] proposed a model of the relationship between mission statements and corporate performance in which top management commitment acts on the relationship between mission statements and corporate performance.

In a study that directly investigated mission statements, Oda et al. [8] analyzed management policies by text mining and reported the following: Management policies can be categorized into three categories, namely, management policies for employees, management policies appealing for social contribution, and management policies with a global perspective. Companies with management policies for employees have the highest performance, whereas companies with management policies appealing for social contribution have the lowest performance. According to Kitajima et al.'s study [9], however, which used text mining similar to Oda et al.'s, manufacturing companies listed on the second section of the TSE, as of October 2013, reported that "companies with a management policy that appeals 
to the world" and "companies with a management policy that appeals to customers" are more profitable. This is in contrast with the results reported by Oda et al.

In addition, because management policies are changed as needed, continuous research is required to extract the relationship between them. In other words, it is implausible that the relationship between management policies and profitability will continue forever, and it is supposed that there are trends in management policies according to specific periods. For example, in a study of manufacturing companies listed on the second section of the TSE, as of 2016, Kitajima et al. [10] reported that the management policies of profitable companies may tend to be described in terms of "customer first." 1

As described above, the relationship between management policy and profitability is still not clear and further research is required. Therefore, in this study, we analyze the management policies of manufacturing companies on the first section of the TSE, as of the end of the 2016 fiscal year.

\section{Methods}

\subsection{Study Flow}

The flow of this study is shown below.

1. Collect management policies and profitability.

2. Create data for analysis by morphological analysis of management policies.

3. Create a classifier that classifies companies with high or low profitability using a neural computational method.

4. Examine the generalization error of the classifier.

5. Interpret the classification result.

The following is a detailed description of the above procedure.

\subsection{Collect Management Policies and Profitability}

In this study, to reduce the time required for collection and ensure the precision of the information, management policies and corporate performance data are obtained from annual securities reports. The merits of using these reports are the accuracy and accessibility of the information. Content from an annual securities report is trustworthy, because, under the Financial Instruments and Exchange Act, the report must be submitted to the Financial Services Agency (FSA, a Japanese government agency), and false statements are illegal. Additionally, these reports are easy to obtain (they can be downloaded from the Electronic Disclosure for Investors' NETwork (EDINET), a service of the FSA). Management policies and profitability information can be obtained from company websites, but as of July 15,2020 , there were 3,717 companies listed on the TSE, the largest financial exchange in Japan; thus, collecting management policies and profitability information from websites is time-consuming.

\footnotetext{
${ }^{1}$ This study reported that words related to "Place - Institution" and "Number" in the management principles may have an impact on profitability, but the results were revised after reanalysis.
} 
In this study, we obtained management policies and profitability from annual securities reports written in the eXtensible Business Reporting Language (XBRL) using XBRL tags as cues. Annual securities reports can be downloaded from EDINET in two file formats: PDF and XBRL. Although an annual securities report in PDF format can be read easily, reports are less readable in the XBRL format; however, XBRL files are easy to process on a computer, and, therefore, we used XBRL format for this research.

The annual securities report includes a section titled "management policy, management environment, issues to be addressed, etc.," and the text in this section was obtained as a candidate for analysis. The management principles to be analyzed were manually extracted from this candidate text. In this study, we decided to analyze manufacturing companies listed in the first section of the TSE. We collected the annual securities reports from 761 companies to extract "management policy, management environment, issues to be addressed, etc." The extracted sentences were visually checked, and the management policies from 699 companies were obtained; whenever possible, we chose policies that were in place since the company's founding, rather than policies relevant to the current corporate environment.

For corporate performance, we focused on profitability and calculated Return On Asset (ROA) as follows.

$$
\mathrm{ROA}=\frac{\text { Ordinary Profit }}{\text { Total Assets }} \times 100 \text {. }
$$

To analyze the performances of individual companies, we obtained and calculated the values of non-consolidated financial results.

\subsection{Create Analysis Data}

Although management policies are collected, as described above, because the data are textual, they are difficult to analyze. Therefore, morphological analysis is performed on the management policies, and the analysis data are created based on the results (using word categories). For morphological analysis and to obtain word categories, we used JUMAN [11], a Japanese morphological analysis system. The categories are the information provided by the JUMAN dictionary, which provides details for words; for example, the word "car" falls under "Category: Artifact - Vehicle." A total of 22 categories are prepared for JUMAN, but in this research, new categories such as "Company name," "Company group name," "Place name: Japan," "Place name: Country," and "Undefined Word" were added; a total of 27 categories were obtained and recorded as TF-IDF values, which were used as analysis data.

For ROA data of the 761 companies whose securities reports we collected, we assigned a target flag of " 0 " (not high performance) to companies in the first quartile (2.2967) or lower and a target flag of "1" (high performance) to companies in the third quartile (7.1187) or higher. For the 699 companies for which we obtained management policies, there were 169 companies for target flag 0 and 180 companies for target flag 1 , resulting in a total of 349 companies for analysis. Although the analysis data composed of 27 input variables, created by the abovementioned method, some categories did not appear in more than half of the data and were subsequently removed. The final analysis data were composed of five variables (the final analysis data variables are shown in Table.1). The data for analysis created by the above work is analyzed by the method described in the next section. 


\subsection{Computational Methods}

The data in this study, composed of management policies and profitability, were complex. Therefore, we decided to use neural networks for the analysis, because they are well known for analyzing complex problems. However, because it is difficult to use neural networks to interpret internal representations, they are referred to as "black boxes." Therefore, we opted to use "potential learning (PL)," a neural network that can interpret internal representations. PL was used by Kitajima et al. for analysis requiring interpretation (for example, analysis of a supermarket [12] and analysis of tweet data [13]), and it has high model performance and high interpretability.

As shown in Figure.1, this method consists of two networks based on self-organizing maps (SOM) and multilayer perceptron (MLP). Figure.1(a) shows the knowledge acquisition step, which calculates the "potentiality" of input neurons and performs knowledge acquisition (learning) based on the SOM. Potentiality is defined as the ability to cope with various situations, and it is regarded as: "neurons with high potentiality (neurons capable of dealing with various situations) are the neurons that play an important role in learning." By interpreting this potentiality after learning is completed, it is possible to interpret the inputs used for learning. Now, let $\phi_{k}^{r}\left(0 \leq \phi_{k}^{r} \leq 1\right)$ denote the potentiality of the $k$ th $(k=1,2, \ldots, K)$ input neuron. This is defined by

$$
\phi_{k}^{r}=\left(\frac{V_{k}}{\max _{k=1, \ldots, K} V_{k}}\right)^{r},
$$

where $V_{k}$ is a variance of the $k$ th input neuron. It can be computed by the connection weights $w_{j, k}$, which denotes the weight from the $k$ th input neuron to the $j$ th output neuron $(j=1,2, \ldots, J)) . r$ is a parameter that controls the computed potentiality. Thus, if parameter $r$ has a large value, then an input neuron with a large variance will have a large potentiality.

After computing potentiality, PL learns in the knowledge acquisition step. In this step, learning is performed by SOM, but potentiality is used as

$$
d_{j}=\sqrt{\sum_{k=1}^{K} \phi_{k}^{r}\left(x_{k}-w_{j, k}\right)^{2}},
$$

in determining the distance $d_{j}$ between the input neuron (the $k$ th input neuron is denoted by $x_{k}$ ) and the $j$ th output neuron. Thus, in the knowledge acquisition step, the distance, weighted by the potentiality of input neurons, is used for learning. Other learning logic is the same standard SOM.

After the knowledge acquisition step, learning in the knowledge utilization step (Figure.1(b)), on which the MLP is based, is performed. In the knowledge utilization step, the

Table 1: List of variables for final analysis data

\begin{tabular}{cl}
\hline No. & \multicolumn{1}{c}{ Name } \\
\hline 1 & Person \\
2 & Organization \\
3 & Artifact - Others \\
4 & Place - Others \\
5 & Abstract things \\
\hline
\end{tabular}




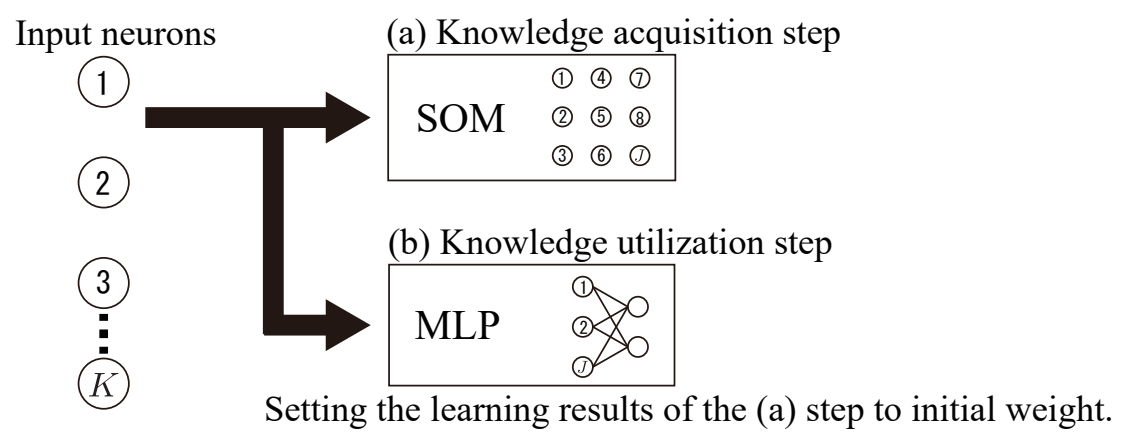

Figure 1: Concept of PL.

initial weights between the input layer and the hidden layer are set to the values calculated from the weights and potentiality, which were obtained in the knowledge acquisition step (weight $\times$ potentiality), and learning is performed. The MLP learning result usually depends on the initial weight; however, PL can learn based on the knowledge acquired by this initial weight setting.

In the next section, we describe the analysis of the data created in the previous section using this PL. In the PL used in this study, the number of input neurons was five; the number of output neurons in the knowledge acquisition step and the number of hidden neurons in the knowledge utilization step was 364 , and there were two output neurons in the knowledge utilization step. The numbers of output neurons in the knowledge acquisition step and hidden neurons in the knowledge utilization step were determined by the analysis software (depending on the setting (mapsize:big) of the SOM Toolbox [14]). Furthermore, in the knowledge utilization step, the transfer function of the hidden neurons was the hyperbolic tangent function, and the transfer function of the output neurons was the softmax function. In addition, the parameter $r$ was changed from 1 to 50 in one step, and the parameter value with the best accuracy rate was identified through a search.

Moreover, the 349 analyzed data were used $70 \%$ for learning, $15 \%$ for overfitting prevention (early stopping), and $15 \%$ for generalization error testing. In addition, with this division ratio unchanged, ten patterns with randomly different data samples were created. Using these patterns, a classifier was developed. This means that ten models were created, and the model performance, including the classification results described in the next section, adopts an average from these ten models.

\section{Results and Discussion}

\subsection{Classification}

As a result of the analysis, we were able to classify the management policies with an accuracy of 0.6115 , a precision of 0.5884 , a recall of 0.6240 , and an F-measure of 0.6032 . At this time, the value of parameter $r$ was 8 . This parameter value occurred when the accuracy was the highest.

As shown in Table.2, the PL results (accuracy, recall, and F-measure) were higher than those obtained by logistic regression analysis (LR), support vector machine (SVM) (C and $\gamma$ of SVM were default settings of MATLAB R2014a used in the analysis), MLP, and PL without potentiality (the network structure is the same as PL, but potentiality unused for 
Table 2: Generalization performance

\begin{tabular}{lrrrr}
\hline \multicolumn{1}{c}{ Methods } & Accuracy & Precision & Recall & F-measure \\
\hline LR & 0.5385 & 0.5512 & 0.6185 & 0.5799 \\
SVM (Linear, $C=1)$ & 0.5385 & 0.5549 & 0.5963 & 0.5710 \\
SVM (RBF, $C=1, \gamma=0.5)$ & 0.5692 & $\mathbf{0 . 5 9 0 8}$ & 0.5704 & 0.5731 \\
MLP & 0.5269 & 0.5130 & 0.5320 & 0.5170 \\
PL without potentiality & 0.5615 & 0.5466 & 0.5640 & 0.5494 \\
PL $(r=8)$ & $\mathbf{0 . 6 1 1 5}$ & 0.5884 & $\mathbf{0 . 6 2 4 0}$ & $\mathbf{0 . 6 0 3 2}$ \\
\hline
\end{tabular}

Table 3: Top 5 appearing words

\begin{tabular}{|c|c|c|c|c|c|}
\hline \multicolumn{3}{|c|}{ Target Flg 0} & \multicolumn{3}{|c|}{ Target Flg 1} \\
\hline Rank & Word & Frequency & Rank & Word & Frequency \\
\hline 1 & Company & 298 & 1 & Company & 365 \\
\hline 2 & Management & 282 & 2 & Management & 288 \\
\hline 3 & Group & 268 & 3 & Group & 258 \\
\hline 4 & Business & 224 & 4 & Business & 191 \\
\hline 5 & Technic & 167 & 4 & Technic & 191 \\
\hline & & & 5 & Principle & 186 \\
\hline
\end{tabular}

training). Thus, it was confirmed that PL was effective in investigating the relationship between management policies and profitability; however, the results show that the relationship is difficult to analyze, because this is not a high-performance result. One reason that the model performance did not improve may be that the volume of data was not large, and the relationship between management policy and corporate performance is moderate. Although the target companies for the analysis were manufacturing companies listed in the first section of the TSE, we were only able to obtain management policies from 699 companies. If the data are expanded to all listed companies (TSE first section, TSE second section, TSE mothers, and so on) or all industries in the first section of the TSE, more data samples can be analyzed. However, the management policy may be different if the listed sections and industries are different [10] [15]. Consequently, it is improbable that analysis across all listed sections or all industries will be appropriate. Therefore, increasing the learning data is not realistic in the analysis.

\subsection{Interpretation}

We now interpret the variables that played a significant role in classification. Figure. 2 shows the potentiality of the input neurons. From this figure, it can be confirmed that the potentiality is the maximum for variable number 5 . This means that variable number 5, which refers to the "Abstract things" category, played a especially important role in classification.

We checked whether variables with high potentiality were the same variables utilized in the network from the viewpoint of weight (Figure.3). The weight between the input layer and the hidden layer of the PL was shown in Figure.3 (c). The square in the figure demonstrates the value of weight; the size of the square indicates the weight value. From this figure, we can observe that large weights are concentrated on the 5th input neuron. According to this result, we conclude that variable number 5 played a important role in classification. Conversely, in MLP (Figure.3 (a)), each weight has a various value in each 


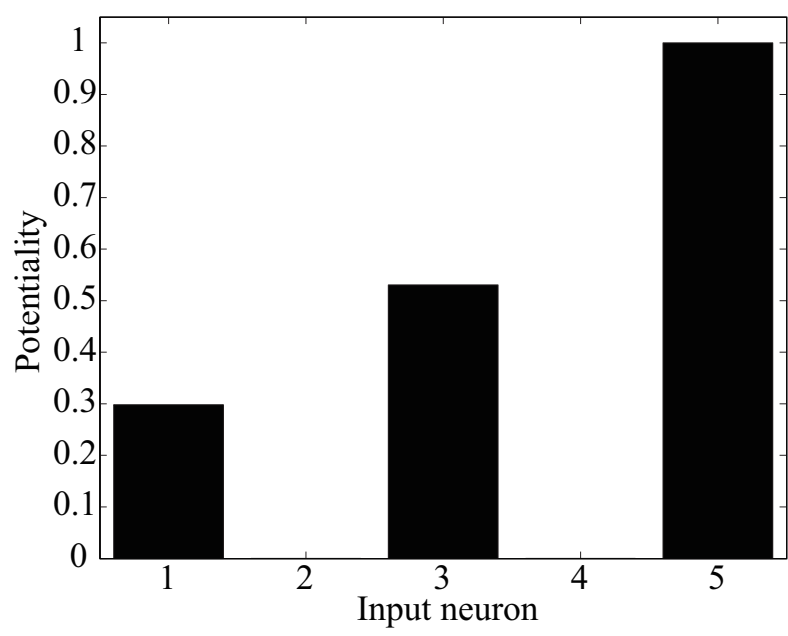

Figure 2: Potentiality of input neurons.

neuron, and it is difficult to interpret the important variables. Although "PL without potentiality" (Figure.3 (b)) seems to be more regular than MLP, it is still difficult to determine which input variables are important.

Now we interpret which words are important from the category "Abstract things." When checking the words in "Abstract things" for each target flag, the total number of occurrences for target flag 0 was 6717 , and the total number of occurrences for target flag 1 was 7153 . The top five occurrences of these words are shown in Table.3. It was confirmed that the word "company" appeared most frequently in both target flags. In addition, there was no difference in the words that appeared in the second rank and below.

Therefore, we conducted a survey of collocations for the word "company," which has the largest number of occurrences; for collocation, we checked the occurrence of the three letters before and after the word. Table.4 shows the top five collocation words for "company," the most frequently occurring word in Table.3. In this table, from left to right, the rank, word, frequency, and the total number of occurrences of the word are displayed. It was confirmed that the same words appeared in the co-occurrences, and it was not possible to find any differences using the number of co-occurrences as a clue. Therefore, we examined the percentage of co-occurrences in the total number of occurrences. We found that there may be a difference in "improvement" (the p-value was the smallest, although no significant difference was obtained (Table.5 shows the results)).

When we checked the specific use of the word "improvement" in the management policy, the descriptions were related to the words shown in Table.6. In companies with target flag 1, for example, descriptions related to "investors," "brand," "governance," and "working environment," were found, which seems to be more diverse than in companies with target flag 0 .

This result may suggest that it is important to include a broad perspective in management policies, such as referring to stakeholders in addition to the company.

\section{Conclusion}

In this study, we analyzed the relationship between management policies and profitability in companies. ROA was used to evaluate profitability, and the management policies were 


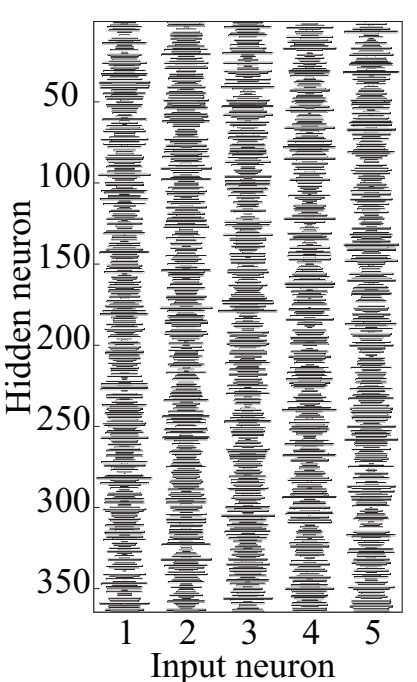

(a) MLP

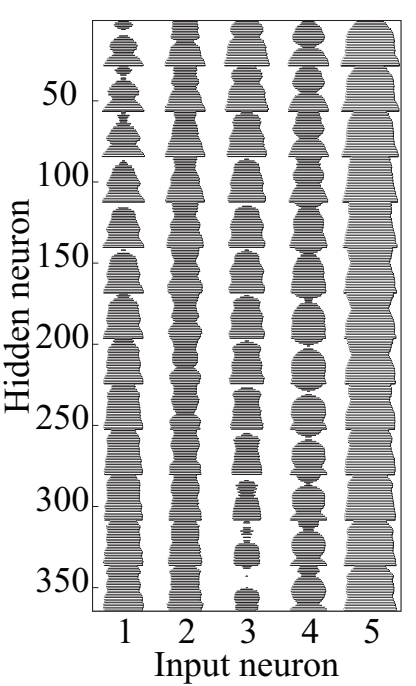

(b) PL without potentiality

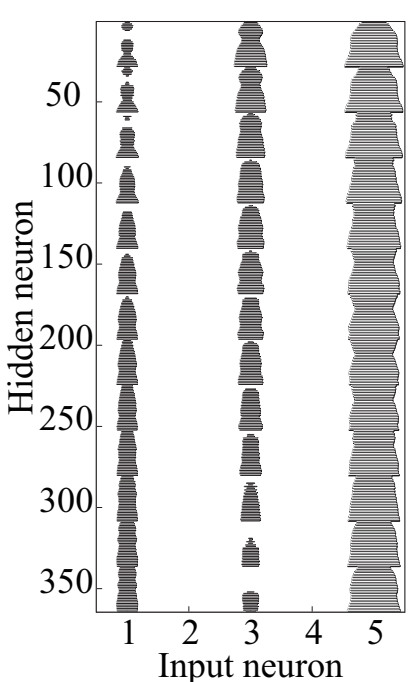

(c) PL

Figure 3: Weights between input layer and hidden layer.

Table 4: Top 5 collocations for "company"

\begin{tabular}{|c|c|c|c|c|c|c|c|}
\hline \multicolumn{4}{|c|}{ Target 0} & \multicolumn{4}{|c|}{ Target 1} \\
\hline \multicolumn{3}{|c|}{ Collocation } & \multirow[t]{2}{*}{ Frequency } & \multicolumn{3}{|c|}{ Collocation } & \multirow[t]{2}{*}{ Frequency } \\
\hline Rank & Word & Frequency & & Rank & Word & Frequency & \\
\hline 1 & Value & 73 & 165 & 1 & Value & 75 & 175 \\
\hline 2 & Principle & 48 & 153 & 2 & Principle & 56 & 186 \\
\hline 3 & Group & 37 & 268 & 3 & Group & 31 & 258 \\
\hline 4 & Improvement & 28 & 110 & 3 & Action & 31 & 83 \\
\hline \multirow[t]{3}{*}{5} & Action & 26 & 85 & 4 & Improvement & 28 & 85 \\
\hline & & & & 4 & Aim & 28 & 114 \\
\hline & & & & 5 & Maximum & 16 & 28 \\
\hline
\end{tabular}

obtained from annual securities reports. Using the annual securities reports as a data source enabled us to obtain reliable data in a short time. Because the relationship between management policies and ROA was complex, we used PL - a neural network that can interpret internal representation - as the analysis method.

The analysis results showed that a model that could classify companies with an accuracy of 0.6115 could be created and that words related to the category of "Abstract things" may influence this classification. We also found that management policies from high-performing companies tend to refer to their stakeholders more than lower-performing companies, which may have influenced the ROA. However, the classification accuracy must be improved, which will be considered in future work. 
Table 5: Result of hypothesis testing for the difference in the population proportions

\begin{tabular}{lc}
\hline \multicolumn{1}{c}{ Word } & $p$ \\
\hline Value & 0.7968 \\
Principle & 0.8016 \\
Group & 0.5406 \\
Improvement & $\mathbf{0 . 2 5 1 9}$ \\
Action & 0.3548 \\
\hline
\end{tabular}

Table 6: Words related to "improvement" and their frequency of occurrence

\begin{tabular}{lrrrr}
\hline \multicolumn{2}{c}{ Target 0} & & \multicolumn{2}{c}{ Target 1 } \\
\cline { 1 - 1 } Related Word & Frequency & & Related Word & Frequency \\
\cline { 1 - 1 } Corporate value & 25 & & Corporate value & 28 \\
Productivity & 8 & & Investors and customers & 3 \\
Culture & 2 & & Management & 3 \\
Life & 1 & & Proceeds & 2 \\
Quality & 1 & & Brand equity & 1 \\
Reliance & 1 & & Governance & 1 \\
Environment & 1 & & Capital efficiency & 1 \\
& 1 & & Productivity & 1 \\
\hline
\end{tabular}

\section{References}

[1] K. Toba and S. Asano, "Sengo nihon no keieirinen to sono henka -keieirinen chousa wo tegakari to shite-," Organizational Science, vol. 18, no. 2, 1984, pp. 37-51 (In Japanese).

[2] Y. Takao, "Are Corporate Creeds Beneficial for Corporate Management?," Management Philosophy, vol. 7, no. 2, 2010, pp. 38-51 (In Japanese).

[3] C. K. Bart and M. C. Baetz, "The Relationship Between Mission Statements and Firm Performance: An Exploratory Study," Journal of Management Studies, vol. 35, no. 6, 1998, pp. 823-853.

[4] J. D. Cort'es-S' anchez, "Mission statements of universities worldwide: text mining and visualization. ," Intangible Capital, vol. 14, no. 4, 2018, pp. 584-603.

[5] K. Nakagawa, S. Sashida, R. Kitajima and H. Sakai, "What Do Good Integrated Reports Tell Us?: An Empirical Study of Japanese Companies Using Text-Mining," Proceedings of the 9th International Congress on Advanced Ap-plied Informatics (IIAI-AAI), 2020, pp. 516-521.

[6] B. S. Patel, L. D. Booker, H. M. Ramos and C. Bart, "Mission statements and performance in non-profit organisations," Corporate Governance, vol. 15, no. 5, 2015, pp.759-774.

[7] R. I. Williams Jr, D. L. Morrell and J. V. Mullane, "Reinvigorating the mission statement through top management commitment," Management Decision, vol. 52, no. 3, 2014, pp. 446-459. 
[8] E. Oda and H. Mitsuhashi, "Mission Statements and Firm Performance: An Empirical Analysis using Text Mining," Management Philosophy, vol. 7, no. 2, 2010, pp. 22-37 (In Japanese).

[9] R. Kitajima, R. Kamimura, and O. Uchida, "Words Which Affect Business Results Included in Mission Statements -From the Point of View of the TF-IDF and Co-occurrence Words-," Proceedings of the 32nd Fuzzy System Sympo-sium, 2016, pp.463-468 (In Japanese).

[10] R. Kitajima, R. Kamimura and H. Sakai, "Relationship Between Management Policies and Profitability for Second Section-Listed Manufacturing Companies of the Tokyo Stock Exchange (2016 Results)," Proceedings of the 8th International Congress on Advanced Applied Informatics (IIAI-AAI), 2019, pp. 697-700.

[11] Kurohashi-Chu-MurawakiLab, Department of Intelligence Science and Technology, Graduate School of Informatics, Kyoto University, JUMAN (A User-Extensible Morphological Analyzer for Japanese) ver.7.0: http://nlp.ist.i.kyoto-u.ac.jp/index.php? JUMAN

[12] R. Kitajima, K. Endou, and R Kamimura, "Creating a Model for Detecting NonContinuous Customers in Retail Stores by Focusing on the Potentiality of Input Neurons," Communication of the Operations Research Society of Japan, vol. 61, no. 2, 2016, pp. 88-96 (In Japanese).

[13] R. Kitajima, R Kamimura, O. Uchida, and F. Toriumi, "Identifying Important Tweets by Considering the Potentiality of Neurons," IEICE Transactions on Fundamentals of Electronics, Communications and Computer Sciences, vol. E99-A, no. 8, 2016, pp. 1555-1559.

[14] J. Vesanto, J. Himberg, E. Alhoniemi and J. Parhankangas, "SOM Toolbox for Matlab 5," Technical report on SOM Toolbox 2.0, 2000.

[15] R. Kitajima and R. Kamimura, "Accumulative Information Enhancement in the SelfOrganizing Maps and Its Application to the Analysis of Mission Statements," Journal of Artificial Intelligence and Soft Computing Research, vol. 5, no. 3, 2015, pp. 161-176. 